\title{
Dementia Research in Australia
}

\section{John McCormack}

The fact that the Alzheimer web page ${ }^{1}$ hosted at Melbourne University has a 'paper of the week' section, in addition to the newsflash section, gives some idea of the pace of research and service development in this area. The November I 995 issue of the Australian Journal of Ageing (vol. 14,4) has five articles on aspects of dementia, one of which is reviewed below, and includes an early paper on the new drug, Risperidone, for those managing agitation among people with dementia. Another aspect of research in this area is its multi-faceted nature, revealing dedicated work on many dimensions of the condition. The recent Australian articles which are reviewed below indicate the scope of current inquiries.

K. Ritchie, C. Mathers and A. Jorm. I 994. Dementia-free life expectancy in Australia. Australian Journal of Public Health, 18 (2), I 49-152.

While disability-free life expectancy has been calculated for the population of Australia, New Zealand and many other countries, this article presents the first estimates of dementia-free life expectancy for Australia. Drawing on the internationally adopted Sullivan methodology, data on the prevalence of dementia, diagnosed by both DSM-I I -R and ICD-io, was collected from a sample of ro45 people aged 70 years and over living in the Australian Capital Territory, and then applied to the I 990 Australian Life Tables. The study derives dementia-free life expectancy data for men and women aged 70-90 years. For those aged 70 years in I990, the mean life expectancy was I 2 years for men and I5.I years for women, while dementia-free life expectancy was II years and I3.7 years respectively. The average life expectancy for those with dementia was only around 0.9 years for men and I.5 years for women. As expected, however, dementia-free years decrease with increasing age, although their ratio to years with dementia is approximately maintained. The results further reveal that the age at which there is the largest absolute number of persons with dementia is around 82 years, which the authors see as an important finding for service development and planning. Another major implication raised by the authors is a complication with the female life expectancy advantage. The female advantage in longevity is associated with higher mental health risks. The authors point out that their results are similar to those of comparable studies in the United Kingdom and 
France, although more longitudinal and culturally comparable work is recommended.
D. Logiudice, W. Woltrowicz, S. McKenzie, D. Ames and L. Flicker. 1995. Prevalence of dementia among patients referred to an aged care assessment team and associated stress in their carers. Australian Journal of Public Health, 19 (3), 275-279.

This study sought to address the large variation in rates of diagnosis of dementia reported by aged care assessment teams (ACATs) across Australia, as well as the condition's implications for carer stress, by applying validated and standardised instruments to a ten per cent random sample of 1000 consecutive referrals to an ACAT at a specialist geriatric hospital in Melbourne. The instruments included the Abbreviated Mental Test Score, the Mini Mental Test Examination, the Cambridge Examination for Mental Disorders in the Elderly, and the Katz Activities of Daily Living (ADL) Scale and the Lawton-Brody Scale of Instrumental ADL. Dementia was diagnosed using ICD- Io, and its severity determined by the five-stage Clinical Dementia Rating Scale. Fifty carers were interviewed using the Zarit Family Burden Scale. The findings of the study revealed a high prevalence $(54 \%)$ of cognitively impaired people. There was also a disproportionately high number of cognitively normal women amongst the referrals. The authors believe the latter finding could be related to the lack of carers for these women. Those suffering dementia also consistently scored lower on physical and instrumental ADL. Carers scored moderately high mean scores on the Zarit Burden test. These scores were not significantly different between those caring for people with severe dementia or for cognitively normal subjects with physical disabilities; both roles are seen as equally difficult for carers. Poor physical function in ADL contributed to carer-burden, while psychological morbidity in carers correlated with personality change and depressive symptoms in the dementia sufferer. The authors plan to follow this cohort to explore which factors among disability, dementia and impaired carer health present the greatest risk factors to elderly people referred to an ACAT.

M. Clark, M. Bond, A. Nankivell and S. Jarrad. I 995. Determinants of the need for respite among carers of people with dementia. Australian Journal on Ageing, 14 (4), 155-159.

In recognition of the fact that many families of dementia sufferers prefer to provide care at home, and that carer strain may increase as 
the disease progresses, this article investigates the determinants and usage of formal and informal respite care and provides an estimate of unmet need. A random sample of 70 carers registered with the South Australian Alzheimer's Association were surveyed using the Strain Scale and the Problem Behaviour Checklist developed by Gilleard $e t$ $a l$., as well as questions specific to unmet need for respite. The results revealed a spread of carer-strain with the highly stressed carers' scores correlating strongly with problem behaviours. However, around half of the carers reported no usage of respite care in the previous twelve months and no problem of unmet need. Among those who had used respite care, equal proportions reported formal or informal care. Situations producing most need for respite included: illness of the carer or cared-for person, the need for a holiday or short break, the wish to participate in social activities, or a need to conduct family business. Multiple regression was used to identify predictors of respite use and unmet need. The three variables retained in the model for usage were: use of informal care, age of the person with dementia, and a female carer. Predictors for unmet need included family illness or other crisis, lack of the carer's confidence that respite would provide adequate care, respite care not catering to a carer's individual needs, and depression about carer's situation. While the study identified two groups of carers - those who rarely used respite, and frequent, regular users - the authors suggest a more active promotion of respite care by formal services and the development of more individualised, flexible services.

\section{COMMENT}

A National Action Plan For Dementia Care (NAPDG) was released in Australia in I99I to consolidate reform in this area and to identify the inadequacies that the Federal government, which has the primary responsibility for aged care, might address. The Plan had emerged from an eight-stage Federal aged-care reform strategy, begun in $1985 / 86$, which has implemented extensive changes in both nursing home and hostel funding, and in staffing standards, service provision targets, a user-rights programme, and the expansion of the home and community care programme. At that stage, services in recognition of the community-wide impact of dementia, more than one billion Australian dollars were provided for aged-care. The Plan set national goals, targets and action over the following five years in seven key areas: diagnosis and assessment, services for people with dementia, services for carers of people with dementia, quality of service, research and evaluation, community awareness, and policy and planning. The 
articles reviewed above have been stimulated by the Plan and are an indicator of the high level of activity in the field.

Despite these developments, sufferers continue to struggle with the day-to-day realities of the disease. Support groups say that even more needs to be done, especially in education for families, their GPs and other health professionals, to prevent families turning to ACATs at a crisis. The Federal government plans a community education programme which should facilitate the implementation of the Plan. Other studies are underway to improve knowledge of how to meet the care needs of people with complex dementias and behaviour. They have also been stimulated by the Plan and are funded from a $\$ 12.4$ million psychogeriatric care and support initiative announced in the 1994/95 Federal budget.

\section{NOTE}

I The World Wide Web address is: 〈http://werple.mira.net.au/ dhs/ad.html〉.

School of Social Work, University of Melbourne

\section{Social Services Expenditure}

\section{Paul Plant}
A. C. Bebbington and A. Kelly. 1995. Expenditure planning in the personal social services: unit costs in the I 980 . Journal of Social Policy, $24(3), 3^{8} 5-41$ I.

Between $1978 / 79$ and $1988 / 89$ there was a 41 per cent real increase in net current expenditure on personal social services in England and Wales, equivalent to a growth rate of 3.5 per cent per annum. Concerns about whether this level of expenditure growth was sufficient has focused on the increased need for social services due to changes in: socio-economic conditions, e.g. the rise in the numbers of unemployed and homelessness; demography, e.g. the increase in the number of the very old; or the responsibility of services, e.g. due to the Children's Act and care in the community. Little work has examined the implications of rising costs on the ability of local authorities to provide services, which the authors of this paper attempt to rectify.

They start by showing how the trends in cost inflation during the rg70s continued into the I980s, to the extent that they more than swallowed up all the expenditure increase, leading to a io per cent reduction in the volume of services provided. Three factors that could 